auparavant : à partir du moment où le lait naturel liquide est remplacé par le lait sec, l'animal montre des signes d'amélioration.

3. Dans les cas où les animaux sont alimentés avec une solution de lait contenant deux fois plus de fer que le lait liquide, la composition chimique du sang de ces animaux n'est pas tout à fait normale. Par contre, celle des rats nourris avec un lait quatre fois plus riche en fer que le lait naturel liquide est analogue à celle des animaux-témoins.

4. L'addition de fer et de cuivre sous forme de combinaisons inorganiques au lait liquide n'est pas aussi efficace que le lait sec.

5. Le rôle du cuivre dans l'anémie expérimentale n'a pas pu être démontré.

6. La teneur élevée en fer du lait sec et d'autres différences dans la constitution chimique, dues au procédé du séchage, semblent avoir des propriétés antianémiques.

\title{
BIBLIOGRAPHIE.
}

[1] Lesné, Clément, Zizin : Le Lait, 11, nº 104, 355 (1931).

[2] Supplee \& Beldis : Journ. Dairy Sci., 5, 455 (1922).

[3] Ch. Porcher : Le Lait desséché. $2^{e}$ édition. Lyon, 1926.

[4] Supplee : Proc. World's Dairy Congress, II, 1248 (1923).

\section{TECHNIQUE D'UNE NOUVELLE RÉACTION POUR DÉCELER LA PASTEURISATION DU LAIT AU-DESSUS DE 58 LEGRÉS}

\author{
par MM. SCHERN et GORLI, \\ de Montevideo, Uruguay.
}

A différentes reprises, des renseignements nous ayant été demandés sur la technique que nous préconisions pour distinguer le lait cru du lait qui avait été chauffé entre 58 et 70 degrés, nous venons, ici, en faire un exposé détaillé.

Jusqu'ici, on pensait le plus généralement, que le lait pasteurisé à basse température, - une demi-heure à $63^{\circ},-$ ou chauffé à une plus haute température, mais sans jamais dépasser $70^{\circ}$, pendant un temps beaucoup plus court, donnait toutes les réactions du lait cru, notamment la réaction de la peroxydase, d'une façon aussi intense que ce dernier. Comme la pratique se rapportait surtout à l'emploi des réactions diastasiques, il en résultait que le lait pasteurisé à basse température ou chauffé un peu plus haut, mais très rapidement, ne pouvait pas être distingué du lait cru.

Notre méthode, dont la technique suit, nous a donné jusqu'à présent des résultats entièrement satisfaisants et, grâce à elle, nous avons toujours pu différencier le lait cru du lait chauffé à 58/60\%. 
Elle est basée sur les modifications que tout liquide biologique subit quand on le chauffe vers $60^{\circ} \mathrm{C}$. Voici en quoi consiste cette technique :

A $1 \mathrm{~cm}^{3}$ de lait, on ajoute une goutte d'une suspension à 1,5\%, dans de l'eau physiologique, de globules rouges de cobaye puisés dans la cuisse de cet animal, ces globules ayant été lavés au préalable, comme cela se fait en sérologie. Les gouttes sont versées, avec une pipette de $1 \mathrm{~cm}^{3}$, qui donne environ 17 gouttes par $\mathrm{cm}^{3}$. Les globules rouges doivent être utilisés de préférence le jour même de leur extraction ou, au plus tard, dans un délai de 48 heures, à condition qu'ils aient été conservés dans un froid sec. S'il y a hémolyse ou si plus de 48 heures se sont écoulées depuis la récolte, il ne faut pas les utiliser.

Pour la réaction, nous nous servons de tubes à essais de $10 \mathrm{~cm}$. de hauteur et de $15 \mathrm{~mm}$. de diamètre environ. On agite bien; puis on porte les tubes au bain-marie, à $38^{\circ}$, pendant 2 heures. Au bout de ce temps, un anneau apparaît dans la zone de la crème, aucun sédiment ne s'est déposé au fond du tube et le lait a gardé sa couleur naturelle. Voilà ce que l'on observe pour le lait cru.

S'il s'agit d'un lait chauffé au-dessus de $58^{\circ}$, c'est-à-dire d'un lait pasteurisé à $63^{\circ} \mathrm{C}$, pendant une demi-heure, on observe un sédiment rouge, assez fin, formant comme un voile et semblable à celui que l'on observe dans la réaction d'hémoagglutination. Quelquefois, il apparaît un anneau rose dans la zone de la crème, mais il est toujours très fin : cet anneau ne peut jamais mettre en doute le résultat de la réaction, le sédiment suffisant à affirmer cette dernière. Avec le lait bouilli, ou chauffé à une température beaucoup plus élevée, le sédiment rouge est plus marqué et forme un véritable disque et, dans ce cas, nous ne notons jamais un anneau rouge ou rose dans la zone de la crème. Rien n'est donc plus simple que la réaction en question, qu'il est facile de réaliser dans un laboratoire.

Pour toutes ces recherches, nous recommandons de faire, avec une partie de l'échantillon du lait, soumis à l'examen, l'épreuve de la teinture de résine de gaiac, ou l'épreuve du gaiacol, de façon à s'assurer tout d'abord que l'on est en présence ou d'un lait chauffé au-dessus de $70^{\circ}$ ou d'un lait qui n'a jamais atteint ladite température; dans ce dernier cas, le lait peut être cru ou pasteurisé à basse température, et c'est ici que notre méthode doit intervenir.

Nous pratiquons alors la réaction avec les globules rouges, qui nous permettra de conclure d'une façon certaine s'il s'agit d'un lait cru ou d'un lait chauffé au-dessus de $58 / 60^{\circ}$, mais au-dessous de $70^{\circ}$.

Nous avons pratiqué cette réaction également avec du lait mouillé jusqu'à $20 \%$ d'eau et la différenciation a toujours été nette. Cette réaction ne convient point avec du lait nettement acide ou avec du lait écrémé. 
Pour les laits écrémés et pour les crèmes, nous recourons à un procédé spécial.

Pour la réaction mentionnée au commencement de ce travail, nous employons aussi de la poudre de charbon animal en suspension à 0,5-1\% dans de l'eau physiologique; les résultats obtenus sont bons, mais pas si bons qu'avec les globules rouges. Les quantités de lait et de suspension sont toujours les mêmes : $1 \mathrm{~cm}^{3}$ de lait et 1 goutte de la suspension de charbon.

Kонn et KLemm, de Carlsbad, ont confirmé nos recherches et ont donné à notre réaction le nom : La réaction de l'Anneau de SchernGorli; ces deux auteurs ont fait les mêmes expériences avec du carmin et de l'indigo et ils ont eu d'aussi bons résultats.

\title{
BIBLIOGRAPHIE
}

Schern et Gordr. - Archivos de la Sociedad de Biologia de Montevideo. Vol. V, $\mathrm{n}^{\circ} 3$, p. $246-49,1930$.

SCHERN et GoRLt. - Berliner Tierärztiche Wochenschrift. 1930, no 47, p. 893.

Конм et Кцемм. - Zeitschrift für Hygiene und Infections-Krankheiten der Haustiere. Tome 39, cahier $1 / 2$.

\section{SUR LE DIAGNOSTIC DU LAIT PASTEURISÉ A BASSE TEMPÉRATURE PAR L'ANNEAU DE SCHERN-GORLI}

\author{
par MM. le Dr F. G. KOHN et Ewald KLEMM, \\ de Carlsbad, République tchécoslovaque.
}

Il n'est pas difficile de reconnaître si un lait a été pasteurisé à des températures élevées, car nous avons à notre disposition des méthodes chimiques et biochimiques simples qui permettent de nous fixer sur ce point.

Au contraire, le diagnostic du lait pasteurisé à basse température présente toujours des difficultés et ce sont ces dernières qui, dans une certaine mesure, ont permis la critique de la pasteurisation à basse température, puisqu'en dehors du dénombrement microbien, nous n'avions aucun procédé sensible et sûr qui pût nous garantir que le lait avait été réellement chauffé à $63^{\circ} \mathrm{C}$. pendant une demi-heure.

Or, le contrôle des laiteries modernes qui travaillent à $63^{\circ} \mathrm{C}$. est indispensable. C'est dire que tout procédé susceptible de nous affirmer qu'il y a eu chauffage à $63^{\circ}$ sera le bienvenu.

Pour ces raisons, nous estimons que la méthode de l'anneau de SCHern-Gorli est du plus grand intérêt. Cette méthode se ramène en somme à l'étude de la direction des mouvements d'une suspension de globules rouges de cobaye dans le lait. Les résultats de leurs expé- 